\title{
Wahrhaftigkeit und Interpretation Der Streit um die wahre Bibelauslegung in Südafrika während der Apartheid ${ }^{1}$
}

Moritz Gräper

\section{Wahrhaftigkeit und Interpretation}

Wahrhaftigkeit kann als Kardinaltugend jeglicher Kommunikation gelten, weil sie einem kommunizierenden Subjekt dazu verhilft, danach zu streben, »die wahrheit zu reden. ${ }^{2}$ Als wahrhaftig kann nämlich nur eine Äusserung gelten, von deren Wahrheit das kommunizierende Subjekt überzeugt ist. Somit schliesst Wahrhaftigkeit zwar keine Fehler und Irrtümer in den Äusserungen ihres Subjekts aus, aber sehr wohl bewusste Täuschungen oder Lügen. ${ }^{3}$ Somit ermöglicht Wahrhaftigkeit in Kommunikationsprozessen Glaubwürdigkeit und Verstehen sowie Austausch und Debatte. »Sie ist die Bedingung der Möglichkeit gelingender Kommunikation [...]. Ohne W[ahrhaftigkeit] kein echtes Gespräch, keine echte Auseinandersetzung. $«^{4}$

Auch im kommunikativen Spezialfall der Interpretation von Texten kommt Wahrhaftigkeit eine wichtige Funktion zu. In der Textexegese begründet eine Haltung der Wahrhaftigkeit die hermeneutische und ethische Redlichkeit des interpretierenden Subjekts, wenn sie auch nicht garantieren kann, dass eine wahrhaftige Auslegung wahr oder ethisch wertvoll ist. Jede Auslegung eines Textes

1 Dieser Aufsatz beruht zu grossen Anteilen auf Erkenntnissen aus meiner von Hermut Löhr (Bonn) und Jeremy Punt (Stellenbosch) betreuten und 2017 in Münster angenommenen Dissertation "Bible and Apartheid. A Hermeneutical and Reception-Historical Study with a Contextual Reading of Matt 15:21-28, « die voraussichtlich in diesem Jahr als Monographie erscheint.

2 Jacob und Wilhelm Grimm, Art. wahrhaftigkeit, in: Deutsches Wörterbuch, Bd. 27, Leipzig 1922, 836-911, hier 836.

3 Dass das Gegenteil von Wahrhaftigkeit die Lüge ist, darin stimmt Eberhard Jüngel, Art. Wahrhaftigkeit/Authentizität. I Fundamentaltheologisch, in: RGG4, Bd. 8, Tübingen 2005, 1242-1243, hier 1242 mit Grimm, wahrhaftigkeit, 836 und anderen Darstellungen des Begriffs überein.

4 Jüngel, Wahrhaftigkeit/Authentizität, 1242. Siehe ebd. auch für Belege der Diskussion über Wahrhaftigkeit als "Grundhaltung des Menschen" und Tugend von der Antike bis in die Gegenwart. 
ist ein Produkt komplexer Kommunikationsprozesse der Rezeption und Produktion zwischen Text und Ausleger_in. Interpretieren führt also zum einen Bedeutung aus einem Objekt hinaus (Exegese) und leitet zum anderen unweigerlich ebenso Bedeutung hinein (Eisegese). Es ist folglich nicht nur bedeutsam, was aus welchem Kontext interpretiert wird, sondern auch wer in welchem Kontext etwas auslegt. Deswegen betont Fernando Segovia zurecht, dass »exegesis ultimately eisegesis" und somit "all interpretation [...] contextual and [thereby] ideological " ${ }^{5}$ ist. Noch zugespitzter bringt Dale Martin die wichtige Rolle des interpretierenden Subjekts auf den Punkt: »Texts don't mean. People mean with texts. ${ }^{6}{ }^{6}$ Innerhalb der Apartheid-Debatte machte der Niederländisch-Reformierte Ethiker Bennie Keet schon früh auf die kontextuelle Verortung der Apartheid-Exegese aufmerksam: "In any case I am convinced that without the background of apartheid as it is practiced in this country we should never have drawn the conclusions we do from our study of the Bible. ${ }^{7}$

Wenn nun die hermeneutisch bedeutsame Rolle der Interpretin oder des Interpreten im Prozess der Auslegung eines Textes zugestanden wird, wird klarer, warum Wahrhaftigkeit in diesem Zusammenhang Gewicht hat. Sie sollte gewissermassen am Ende eines jeden erfolgreichen hermeneutischen Prozesses stehen, indem die interpretatorische Äusserung mit den Überzeugungen ihres Subjekts über die Wahrheit derselben übereinstimmt.

Gleichzeitig eröffnet jede Konfrontation mit einem Text eine zweifache ethische Dimension der Interpretation: "Jedes Lesen ist ein Interpretieren, jedes Interpretieren ist ein Handeln, ein SichVerhalten $\mathrm{zu}$ einem Anderen, Vorgegebenen. Gelesen wird ETWAS, ein Anderes, das man nicht selbst ist. [...] Die Rezeption der Äußerung eines anderen ist nicht nur ein hermeneutisches, sondern grundlegend immer auch ein ethisches Problem. Wie verhalte ich

\footnotetext{
5 Fernando F. Segovia, My Personal Voice. The Making of a Postcolonial Critic, in: The Personal Voice in Biblical Interpretation, hg. v. Ingrid Rosa Kitzberger, London 1999, 25-37, hier 31. »Ideological« mag für manche in diesem Kontext befremdlich klingen. Versteht man unter Ideologie allerdings die Summe aus Prägungen, Überzeugungen und Werten eines Menschen, die er an einen von ihm auszulegenden Text mit heranträgt, macht der Ausdruck hier durchaus Sinn.

${ }^{6}$ Dale B. Martin, Sex and the Single Savior. Gender and Sexuality in Biblical Interpretation. Louisville, KY 2006, 1.Vgl. die ausführliche Diskussion um die Rolle von Autor, Text und Leser in der Interpretation von Texten im Theorie- und Methodenkapitel von Gräper, Bible and Apartheid.

7 Bennie Keet, Whither-South Africa, Stellenbosch 1956, 19.
} 
mich zu einem Anderen? « ${ }^{8}$ Eberhard Jüngel qualifiziert Wahrhaftigkeit zutreffend als Relationsbegriff, der die Übereinstimmung der Lebensäusserung eines Menschen im Verhältnis zu sich, seinen Mitmenschen und Gott mit dem von ihm für wahr gehaltenem beschreibt. ${ }^{9}$ Angesichts der ethischen Dimension des Interpretierens müsste diese Beschreibung um ein viertes Verhältnis der potentiellen Wahrhaftigkeit, nämlich dem zwischen Mensch und Text, erweitert werden. Ethische Verantwortlichkeit fordert allerdings nicht nur der Text als fremdes Gegenüber, sondern auch der zeitgenössische Kontext des Exegeten oder der Exegetin. Dies ist insbesondere der Fall mit Blick auf die Auslegungsgeschichte der Bibel, die mehr als genügend Beispiele für aus heutiger Sicht missbräuchliche Interpretationen bietet. Man denke nur an die Rechtfertigung der Sklaverei in den USA $^{10}$, die antisemitische Exegese in Nazi-Deutschland ${ }^{11}$ oder die Legitimierung der Apartheid mit Hilfe von Bibelauslegungen in Südafrika, ${ }^{12}$ die uns im Folgenden als Fallbeispiel für die ethische Herausforderung von Bibelinterpretationen dienen wird.

Elisabeth Schüssler Fiorenza fordert seit ihrer Presidential Address bei der Jahrestagung der Society of Biblical Literature von 1987 eine diesen Befund ernst nehmende Ethik der Interpretation in der Bibelwissenschaft, die dieser doppelten ethischen Anforderung gerecht wird:

"If the Bible has become a classic of Western culture because of its normativity, then the responsibility of the biblical scholar cannot be restricted to giving sthe readers of our time clear access to the original intentions of the biblical writers. It must also include the elucidation of the ethical consequences and political functions of biblical texts in their historical as well as in their contemporary sociopolitical contexts. $\ll^{13}$

\footnotetext{
8 Stefan Alkier, Ethik der Interpretation, in: Der eine Gott und die Welt der Religionen. Beiträge zu einer Theologie der Religionen und zum interreligiösen Dialog, hg. v. Markus Witte, Würzburg 2003, 21-41, hier 21.

9 Vgl. Jüngel, Wahrhaftigkeit/Authentizität, $1242 \mathrm{f}$.

${ }^{10}$ Vgl. z.B. J. Albert Harrill, Slaves in the New Testament. Literary, Social, and Moral Dimensions, Minneapolis, MN 2006, 165-196.

${ }_{11}$ Vgl. z.B. Susannah Heschel, The Aryan Jesus. Christian Theologians and the Bible in Nazi Germany, Princeton, NJ 2008.

${ }^{12}$ Vgl. z.B. Bobby Loubser, The Apartheid Bible. A Critical Review of Racial Theology in South Africa, Lewiston, NY 1987; Dirkie Smit, Art. Apartheid, in:The Oxford Encyclopedia of the Bible and Ethics, London 21-29; Gräper, Bible and Apartheid.

${ }^{13}$ Elisabeth Schüssler Fiorenza, The Ethics of Biblical Interpretation: Decentering Biblical Scholarship, in: Journal of Biblical Literature 107 (1988), 3-17, hier 15.
} 
So erfordert die Situation des Auslegens von dem Exegeten oder der Exegetin, dass er oder sie sich angesichts dieser ethischen Verantwortung gegenüber Text und Kontext verhält. Eine mögliche und meines Erachtens anzustrebende Haltung ist die der Wahrhaftigkeit gegenüber Text und Kontext, weil diese den Text nicht missbraucht oder den Kontext manipuliert, sondern so weit wie möglich offenlegt, warum und inwiefern die Interpretation des Textes und Äusserung in einen bestimmten Kontext hinein mit dem inneren Fürwahrhalten des Auslegers oder der Auslegerin übereinstimmt. Zustimmung, Kritik und Diskussion werden durch Wahrhaftigkeit in der Interpretation ermöglicht, da wahrhaftige Äusserungen sich selbst nicht verdecken, verschleiern oder verkleiden, sondern sich möglichst transparent mitteilen.

\section{Der theologische Apartheiddiskurs}

Aus theologischer und speziell hermeneutischer Sicht ist die Geschichte der Apartheid ein einzigartig dichtes Forschungsfeld. Der reformierte Theologe Allan Boesak formulierte bereits 1984 pointiert:

"Apartheid is unique. But its uniqueness does not lie in the inherent violence of the system [...]. No, the uniqueness of apartheid lies in the fact that this system claims to be based on Christian principles. It is justified on the basis of the gospel of Jesus Christ. It is in the name of the liberator God and Jesus Christ, the Son of God, that apartheid is perpetuated, and it is Reformed Christians who are responsible for it. ${ }^{14}$

Es ist historisch zutreffend, christlichen Akteuren die Verantwortung für die Apartheid zuzuschreiben. »It was not the state but the church who took the lead with apartheid. The state followed the principle laid down by the church [...]. ${ }^{15}$ Zusätzlich muss bedacht werden, dass seit der politischen Einfuihrung der Apartheid 1947, » the DRC and the state had become essentially one. ${ }^{16}$

\footnotetext{
${ }^{14}$ Allan Aubrey Boesak, Black and Reformed. Apartheid, Liberation and the Calvinist Tradition, Maryknoll, NY 1990, 85.

${ }^{15}$ Der erste Apartheid-Premierminister Daniel François Malan zitiert in Hermann Giliomee, The Afrikaners. Biography of a People, London 2011, 460.

${ }^{16}$ Jacobus Christoff Pauw, Anti-Apartheid Theology in the Dutch Reformed Family of Churches, online-publizierte Dissertation an der Freien Universität Amsterdam 2007, 115. Zu nennen ist insbesondere der geheime »Broederbond«. Dieser vernetzte
} 
Ein komplexes Geflecht ${ }^{17}$ aus 1. kolonialistischen und rassistischen Denkmustern, die mit den europäischen Siedlern am Kap der guten Hoffnung landeten, 2. der Erfahrung von politischer und kultureller Marginalisierung durch das übermächtige British Empire, die in den sogenannten Anglo-Boer-Wars und den Konzentrationslagern für die afrikaansen Siedler ihren traurigen Höhepunkt fanden, 3. neocalvinistisch-philosophischem Denken im Anschluss an Abraham Kuyper, das durch die hohe Anzahl der Niederländisch-Reformierten Auslandstudenten und -promovenden in Amsterdam nach Südafrika importiert wurde sowie 4. der Rezeption des vom deutschen Missionstheologen Gustav Warneck geprägten Begriffs Volkschristianisierung stellt die wichtigsten Aspekte dar, um verstehen zu können, wie vor allem afrikaanse Theologen ab den 1930er Jahren durch ihre Bibelinterpretationen zu dem Schluss kommen konnten, dass Gott selbst die Apartheid rechtfertigt. ${ }^{18}$

Die erste kirchenpolitische Zäsur, die die Separationsideologie des späteren Apartheidregimes der Nationalen Partei ab 1948 in gewisser Weise vorwegnahm, bildet eine Synodenentscheidung der Niederländisch-Reformierten Kirche aus dem Jahr 1857, die verfügte, dass "aufgrund der Schwachheit von einigen" (ten gevolge van de zwakheid van sommigen $)^{19}$ nach Hautfarbe getrennte Abendmahlsfeiern erlaubt seien. Zwar wurde dieser Entschluss nicht mit dem Prädikat "nach Gottes Willen« o.ä. ausgestattet, sondern ausdrücklich als pragmatische Notlösung gefasst. Jedoch entstanden in der Folge die durch ethnische Grenzen strukturierte NiederländischReformierte "Kirchenfamilie," die separate Kirchen für Schwarze,

ab 1921 afrikaans-nationalistische Pastoren, Politiker und Akademiker.Vgl. T. Dunbar Moodie, The Rise of Afrikanerdom. Power, Apartheid, and the Afrikaner Civil Religion, Berkeley, CA 1975.

${ }_{17}$ Vgl. John W. de Gruchy, The Church Struggle in South Africa. Twenty-Fifth Anniversary Edition with Steve de Gruchy, London 2004 [1979] und das Kapitel »Trajectories towards Apartheid" in Gräper, Bible and Apartheid für zusammenfassende Darstellungen der in diesem Aufsatz nur angerissenen Aspekte, die der Apartheid den Weg ebneten. Eine beeindruckend kenntnis- und quellenreiche Darstellung und Analyse der Geschichte der Apartheid anhand der Historie des afrikaansen Volkes bietet Giliomee, The Afrikaners.

${ }_{18}$ Vgl. z.B. Geoffrey Cronjé (Hg.), Regverdige rasse-apartheid, Stellenbosch 1947. Vgl. ferner die detaillierte hermeneutische Analyse der acht wichtigsten biblischbegründeten Pro-Apartheid-Dokumente im Kapitel "Legitimizing Apartheid with Scripture in Gräper, Bible and Apartheid.

19 Vgl. Smit, Apartheid, 25. 
Farbige $^{20}$, Asiaten und Weisse ${ }^{21}$ institutionalisierte. ${ }^{22}$ Diese Trennung wurde bis in die 1980er Jahre hinein nach innen und aussen mit einem sicher ernst gemeinten und doch aus heutiger Sicht als euphemistisch zu bezeichnenden Sprachgebrauch begründet: Es gehe nicht um rassistisch motivierte Trennung. ${ }^{23}$ Vielmehr ermögliche die ethnische Separation die bestmögliche "getrennte« (aparte) oder wautochthone Entwicklung" (eiesoortige ontwikkeling) einer jeden Ethnie gemäss ihrem kulturellen Entwicklungsniveau im Geiste der Nächstenliebe und mit dem "weissen Volk" als "Vormund" (voog) für die Anderen: ${ }^{24}$ "A political system based on the autogenous or separate development of various population groups can be justified

${ }^{20}$ Die Gruppe der "Farbigen« (Coloureds) wird oft als "Mischlinge« aus Verbindungen von Weissen und Schwarzen übersetzt. Dieses Verständnis trifft auf einige, aber bei weitem nicht alle Coloureds zu, denn mehrheitlich sind Farbige Nachfahren von KhoiSan und deportierten ostasiatischen Sklaven. Vgl. Christoph Marx, Südafrika. Geschichte und Gegenwart, Stuttgart 2012, 225.

${ }^{21}$ Der offensichtlich pseudowissenschaftliche Charakter dieser "Rasseneinteilungen" wird sehr scharf analysiert von Deborah Posel, The Apartheid Project, 1948-1970, in: The Cambridge History of South Africa, Bd. 2: 1885-1994, hg. v. Robert Ross, Anne K. Mager und Bill Nasson, Cambridge 2011, 319-368, hier 333f.

${ }^{22}$ Vgl. "South African Churches and Ecumenical Bodies during Apartheid« in Gräper, Bible and Apartheid.

${ }^{23}$ Besonders aus ihren Apologien kann das Selbstverständnis der Apartheid-Befürworter herausgelesen werde.Vgl. Willem Adolf Landman,A Plea for Understanding. A Reply to the Reformed Church in America. Kapstadt 1968. Der Vorwurf des Rassismus oder gelegentliche Vergleiche mit nationalsozialistischer Ideologie wurden stets emphatisch zurückgewiesen. Karl Barth z.B. bezeichnete die Apartheidtheologie jedoch ausrücklich als "Nazi-Theologie."Vgl. Ben Marais, Colour. Unsolved Problem of the West, 309. Auf meiner Kopie eines frühen Anti-Apartheid-Aufsatzes der Vorgänger-Organisation des South African Council of Churches (Christian Council of South Africa, Race-What does the Bible say? Report of a Commission Appointed by the Christian Council of South Africa to Investigate the Teaching of the Bible on Race Questions, Rodepoort 1952) aus dem Magazin-Bestand der Universitätsbibliothek Stellenbosch findet sich die wahrscheinlich aus den 1950er Jahren stammende Notiz am Rand: 'NO! We are no NAZIS.r

${ }^{24}$ Vgl. z.B. W. J. van der Merwe, Segregasie en Aparte Woongebiede, in: Op die horison 4 (1942), 15-22; A. B. du Preez, Eiesoortige Ontwikkeling tot Volksdiens, Kapstadt 1959 und Dutch Reformed Church, Kerk en Samelewing. 'n Getuienis van die Ned Geref Kerk Uitgegee deur die Algemene Sinodale Kommissie, Bloomfontein 1990, 47. Dieses Apartheidverständnis kommt dem zentralen Narrativ der sogenannten Identitären Bewegung nahe, das den "'Ethnopluralismus`, dem zufolge jedes Volk einen bestimmbaren Wesenskern und einen historisch zugewiesenen (Wirkungs-) Raum habe" propagiert. Martin Eimermacher/Christian Fuchs/Paul Middelhoff, Ein aktives Netzwerk. Um die AfD scharen sich Dutzende Denkfabriken, Zeitschriften und Stiftungen, Die Zeit 45 (2017), 45, http://www.zeit.de/2017/45/afd-netzwerkzeitschriften-stiftungen-verlage (21.11.2017). 
from the Bible, but to love one's neighbor must at all times be the ethical norm towards establishing sound inter-people relations. ${ }^{25}$ Dass Apartheid de facto immer ein Instrument zur Stabilisierung weisser Privilegien war und exklusiv der "white supremacy ${ }^{26}$ diente, wurde früh erkannt:

»The important thing to remember about Apartheid, segregation or what you will, is that it is designed to keep the black man as cheap labour to serve the white man's needs while ensuring that all wealth, prestige symbols and political power are effectively held in the white man's own capable hands. All other arguments, like sdevelopment along their own liness, separate development etc., are just as much eyewash for a policy that has nothing to commend it but the rapacity of the white man $2^{27}$

Die theologisch-motivierten Stimmen, die den status quo in Südafrika kritisierten, werden ab den 1950er Jahren lauter und sind sich spätestens ab den 1970er Jahren einig mit internationalen ökumenischen Organen, dass Apartheid theologisch als Häresie zu verurteilen ist. ${ }^{28}$ Oft wurden zur Widerlegung der biblischen (und damit für die meisten damaligen Theologen auch göttlichen) Legitimation der Apartheid dieselben biblischen Texte bemüht, die auch die Gegenseite in Anschlag gebracht hatte, um interpretatorisch den gegenteiligen Schluss zu ziehen. Plastisch wird dieses hermeneutische Dilemma an der Auslegungsgeschichte des wichtigsten Verses der Apartheiddebatte Apg 17,26. ${ }^{29}$ Während der erste Versteil als Beleg für die schöpfungstheologisch begründete Einheit und Gleichheit aller Menschen und damit gegen die Separation verschiedener Völker gelesen wurde (»Und er hat aus einem Menschen das ganze

${ }^{25}$ Dutch Reformed Church, Human Relations and the South African Scene in the Light of Scripture. Official translation of the Report «as, Volk en Nasie en Volkereverhoudinge in die lig van die Skrif' Approved and Accepted by the General Synod of the Dutch Reformed Church, Pretoria 1976.Vgl. bereits Evert Philippus Groenewald, Apartheid en Voogdyskap in die Lig van die Heilige Skrif, in: Regverdige rasseapartheid, hg. v. Geoffrey Cronjé, Stellenbosch 1947, 40-67, für den Zusammenhang von Apartheid und Nächstenliebe.

${ }^{26}$ South African Council of Churches, A Message to the People of South Africa, Braamfontein 1968, 4.

${ }^{27}$ Nimrod Mkele, Domesticating the African, unpublished 1959, quoted in Peter Randall, A Taste of Power, Johannesburg 1973, 13.

${ }^{28}$ Vgl. de Gruchy, Church Struggle, 187-204.

${ }^{29}$ In meiner Dissertation konnte ich zeigen, dass die weit verbreitete Ansicht, dass alttestamentliche Texte wichtiger für die Apartheiddebatte waren als neutestamentliche nicht von den Quellen gedeckt ist, sondern das Gegenteil der Fall ist. Apg 17,26 wird in nahezu allen relevanten Dokumenten zitiert und ausgelegt. 
Menschengeschlecht gemacht, damit sie auf dem ganzen Erdboden wohnen.«), verkauften die Befürworter "getrennter Entwicklung" den zweiten Versteil als Apartheid in a nutshell ("Und er hat festgesetzt, wie lange sie bestehen und in welchen Grenzen sie wohnen sollen.").

So wird die Apartheid aus bibelwissenschaftlicher Sicht ein "major test case" und geradezu ein »thought-laboratory hermeneutics. $\mathbb{1}^{30}$ Die hohe politische Relevanz der kontroversen Beiträge zum biblisch-theologischen Apartheiddiskurs brachte mit sich, dass in vielen veröffentlichten Bibelauslegungen der Zeit ein hohes Mass an Überzeugung von der Wahrheit der eigenen Interpretation sichtbar wird. Insofern können viele Akteure des Streits um die wahre Bibelauslegung der Apartheid als wahrhaftige Exegeten bezeichnet werden.

\section{„Wahrhaftige« Bibelauslegungen}

"Zeige mir einen Text, « sagt der Gegner unserer Rassenpolitik, reinen Text, der beweist, dass Apartheid im Einklang mit der Botschaft der Heiligen Schrift ist. \& Ich habe keinen Text, < ist meine Antwort. >Dann habe ich den Streit für mich entschieden, s sagt der Gegner erleichtert. IIch habe einen Text: In Kol 3,11 steht: Da ist nicht mehr Grieche oder Jude, Beschnittener oder Unbeschnittener, Nichtgrieche, Skythe, Sklave, Freier, sondern alles und in allen Christus.< Ich entgegne: >Das ist kein Beleg. Ich habe vielleicht keinen einzelnen Text, aber ich habe die Bibel, die ganze Bibel. Meine Argumente werden aus Versen von der Genesis bis zur Offenbarung des Johannes gebildet.: ${ }^{31}$

Dieser stilisierte Dialog eines Befürworters mit einem Gegner der Apartheid eröffnet den 1944 vor dem sogenannten "Volkskongress" gehaltenen Vortrag vom populären afrikaansen Theologen und Poeten Jacob Daniël du Toit, der unter seinem Künstlernamen Totius grosse Berühmtheit erlangte. Und in der Tat setzt er im Verlauf seines Vortrags den Anspruch um, Verse und Perikopen aus dem Alten

\footnotetext{
${ }^{30}$ Richard Burridge, Imitating Jesus. An Inclusive Approach to New Testament Ethics 2007, 350.Vgl. für dieselbe Ausdrucksweise Dirkie Smits Vorwort für Bernard Lategan, Hermeneutics and Transformation. Selected Essays, Stellenbosch 2015, 3-13. ${ }^{31}$ Jacob Daniël du Toit, Die Godsdienstige Grondslag van ons Rassebeleid, in: Die Afrikaanse Rassebeleid en die Skrif, hg. v. Stephan du Toit, Potcheftstroom 1955, 5-21, hier 5. Diese wie alle anderen Übersetzungen aus afrikaansen Quellen habe ich mit Unterstützung von der namibischen Theologin Anneliese Hofmann angefertigt.
} 
und Neuen Testament zusammenzutragen, um seine These von der biblisch und damit göttlich begründeten Apartheid durchzugehen. ${ }^{32}$ Auch Texte wie der eingangs zitierte Abschnitt Vers Kol 3,11 oder der ähnlich klingende Intertext Gal 3,28 werden im Sinne der Apartheidideologie gedeutet, indem die verkündete Einheit in Christus spiritualisiert verstanden wird, während die Aufrechterhaltung "natürlicher" Unterscheidungsmerkmale wie Ethnie, Sprache, Kultur und Geschlecht als selbstverständlich auch aus diesen Bibelversen begründbar dargestellt werden. ${ }^{33}$ Totius scheut in seinem Vortrag nicht davor zurück, einige seiner Grundüberzeugungen offen zu legen. Insbesondere seine Wertschätzung für »sein« Volk, was beim sogenannten Great Trek "geboren ${ }^{34}$ und mit einer besonderen Berufung ausgestattet worden sei sowie seine abschätzige Sicht auf Schwarze werden im Verlauf seiner Ausführungen deutlich: "Sie repräsentieren einen seltsamen Typus innerhalb der Menschheit.< [...] Wir wollen keine Gleichstellung [gelykstelling] und keine Bastardisierung [verbastering] ${ }^{35}$ Auch wenn die rassistische und menschenverachtende Rhetorik von du Toit aus heutiger Sicht natürlich befremdet und kritisch im damaligen Kontext zu beurteilen ist, kann seine Bibelauslegung nicht als unwahrhaftig markiert werden. Im Gegenteil: Totius zeigt eine ganz deutliche Übereinstimmung seines inneren Fürwahrhaltens mit seinen Äusserungen nach aussen. Er benennt gleichzeitig zwei der prototypischen existentiellen Ängste des afrikaansen Volkes, die schon vor Einführung der Apartheid die Separationsobsession angesichts der numerischen Überlegenheit der umgebenden Völker nährten. ${ }^{36}$ Die Angst als weisse, Afrikaanssprechende, kulturell unterscheidbare Entität unterzugehen und zu einer $»$ kaffeefarbenen Bastardbevölkerung ${ }^{37} \mathrm{zu}$ werden, scheint viele Bibelinterpretationen der Zeit zu determinieren.

Als durch innenpolitische Zäsuren wie das Sharpeville-Massaker (1960) oder das Soweto-Uprising (1976) die mörderische Brutalität des Apartheid-Regimes offen zu Tage tritt und die nationale und internationale Kritik wächst, werden auch die biblischen Legitimie-

\footnotetext{
${ }^{32}$ Er bezieht sich im Laufe seines Vortrages auf mehr als 30 Bibelverse oder -perikopen aus Gen, Lev, Dtn, Mt, Apg, Röm, 1Kor, Gal, Kol und Offb.

${ }^{33}$ Vgl. du Toit, Ons Rassebeleid, 9f. Diese interpretatorische Strategie prägt beinahe alle Pro-Apartheid-Dokumente.

${ }^{34}$ Ebd., 6f.

35 Ebd., 13-15.

${ }^{36}$ Vgl. Giliomee, The Afrikaners, 88f.

${ }^{37}$ J. G. (Valie) Strydom, Die Rasse-Vraagstuk in Suid-Afrika, in: Koers in die Krisis, Bd. 3, hg. v. Federasie van die Calvinistiese Studen-tenverenigings in Suid-Afrika, Stellenbosch 1941, 243-255, hier 246.
} 
rungsversuche der Apartheid etwas zurückhaltender in ihrer Sprache. 1974 versuchte die Niederländisch-Reformierte Kirche ein letztes Mal in einem offiziellen Dokument den status quo theologisch zu rechtfertigen. Die vergleichsweise verklausulierte Sprache kann die Verunsicherung und die gewachsene innerkirchliche Kontroverse über die Gesellschaftsordnung nicht verbergen:

"Man darf schlussfolgern, dass das Neue Testament die Möglichkeit vorsieht, dass ein bestimmtes Land seine zwischenmenschlichen Beziehungen unter Beachtung seiner eigenen speziellen Umstände unter Berücksichtigung der Grundnormen, die die Bibel für die Gestaltung sozialer Beziehungen vorschreibt, auf der Basis von getrennter Entwicklung regulieren kann. ${ }^{38}$

Es scheint fast so, als wäre die Reduktion der inneren Überzeugung von der Richtigkeit der Äusserung in dem offiziellen Kirchendokument hier greifbar. Damit wäre dies ein Beispiel für eine wenig wahrhaftige Interpretation. Jedenfalls klingt diese Formulierung ganz anders als die oben zitierten Ausführungen von Totius oder das folgende exegetische Fazit des einflussreichen Neutestamentlers Evert Philippus Groenewald von 1947: „Der Herr will, dass getrennte Völker ihre Apartheid beibehalten. ${ }^{39}$

Während der zu vernehmende Grad der Überzeugung von der Wahrheit der eigenen Interpretation der Bibel hinsichtlich der Frage der Apartheid auf der legitimierenden Seite mit der Zeit abnimmt, intensiviert dieser sich bei den Anti-Apartheid-Theologen zunehmend. ${ }^{40} 1952$ wagt Ben Marais, ein Niederländisch-Reformierter Pastor und Theologieprofessor, die biblische Grundlage der politischen Theologie seiner eigenen Kirche zu negieren: "Any policy of conscious racial segregation in the Christian Church [...] rests on very frail grounds, as far as Scripture is concerned. The Bible definitely gives no such stipulation, and it is even doubtful to what extent it is even permitted by Scripture. $\|^{41}$ Trotzdem befürwortete er aus "weighty practical «2 Gründen die Apartheid für Südafrika, denn "racial crossing, where there is great difference in type $[\ldots]$ as it is the case in our own country, $[\ldots]$ is for a variety of reasons extremely undesirable and dangerous, and I wish to disapprove of it unequivocally. «3 $^{43}$ Einige typische Apartheid-Interpretationen, wie

\footnotetext{
${ }^{38}$ Dutch Reformed Church, Human Relations, 32.

${ }^{39}$ Groenewald, Apartheid en Voogdyskap, 47.

${ }^{40}$ Vgl. das Kapitel "Opposing Apartheid with Scripture» in Gräper, Bible and Apartheid.

${ }^{41}$ Marais, Colour, 292.

${ }^{42}$ Ebd.

${ }^{43}$ Ebd., 295.
} 
die spiritualisierenden Lesarten der Einheit in Christus von Kol 3,11 bzw. Gal 3,28, werden schon früh angegriffen: »A spiritual unity, however, that exists only in the imagination and does not reveal itself in the tangible reality of our daily lives is no true unity; its genuineness can only be tested by putting it into practice. ${ }^{44}$

Kurz nachdem der Reformierte Weltbund bei seiner Sitzung in Ottawa angesichts der Situation in Südafrika einen status confessionis deklariert, den schwarzen Reformierten Anti-Apartheid-Theologen Allan A. Boesak zum Präsidenten gewählt und die zwei weissen Niederländisch-Reformierten Kirchen aus Südafrika aus der ökumenischen Gemeinschaft suspendiert hatte, entsteht 1982 das kontroverse Belhar-Bekenntnis. In der Tradition der Message to the People of South Africa von 1968 verurteilte Belhar die Apartheid theologisch als falsche Lehre und Bedrohung des Evangeliums. ${ }^{45}$ Das Widerstandsbekenntnis weist viele Analogien zur Barmer TheologischenErklärung auf. ${ }^{46}$ Es lässt keinen Zweifel daran, dass es den status quo in Südafrika für biblisch unbegründet und damit für theologisch für falsch hält: "We believe that any teaching which attempts to legitimate such forced separation by appeal to the gospel [...] must be considered ideology and false doctrine. ${ }^{47}$ Sie reklamieren die wahre Interpretation der Bibel für sich, genauso wie es vor ihnen die ProApartheid-Theologen auch getan hatten. Und doch unterscheiden sie sich nicht nur inhaltlich von diesen, indem sie einräumen, dass es gefährlich sei, die eigenen Auslegungen als wahre Botschaft Gottes auszugeben, da Menschen immer irren könnten. Sie tun es aber trotzdem, da sie selbst von der Wahrheit überzeugt seien. Insofern machen die Autoren von Belhar eine Haltung der Wahrhaftigkeit im vollen Bewusstsein der Irrtumsanfälligkeit menschlicher Interpretationen für sich geltend. ${ }^{48}$

\footnotetext{
${ }_{44}$ Bennie Keet, Whither-South Africa, 27.

${ }^{45}$ Piet Naudé, Piet. Neither Calendar Nor Clock. Perspectives on the Belhar Confession. Grand Rapids, MI 2010, 3.

${ }^{46}$ Vgl. z.B. Evangelisch-reformierte Kirche/Lippische Landeskirche/Reformierter Bund in Deutschland (Hg.), Für das Recht streiten. 30 Jahre Bekenntnis von Belhar. Texte und Anregungen, Hannover 2016, http://www.reformiert-info.de/15410-0-01.html (21.11.2017).

47 Naudé, Belhar-Confession, 221f.

${ }^{48}$ Naudé, Belhar-Confession, 3. Fraglich ist, ob es als "wahrhaftig« gelten kann, keine Motivation - ausser der Entfaltung der wahren Bedeutung des Wortes Gottes Raum zu geben - anzuführen und zu meinen, keine "group interests $z$ zu vertreten. Belhar vertritt m.E. offensichtlich ein starkes (und natürlich legitimes) Gruppeninteresse der theologischen Apartheidgegner. Die Behauptung des Gegenteils scheint mit der theologischen Fantasie der Eindeutigkeit des Wortes Gottes zusammenzuhängen.
} 
Das international wirkmächtigste Dokument des theologischen Widerstands gegen die Apartheid wird drei Jahre nach dem BelharBekenntnis von einer Gruppe von Theologen verschiedener Konfessionen in Soweto verfasst. Das Kairos-Dokument trägt die eigene Haltung der Wahrhaftigkeit im Titel: Der neutestamentliche Begriff xaıpós wird mit "moment of truth" übersetzt. Dieser Moment der Wahrheit wird als Zeitpunkt der Herausforderung durch Gott verstanden, sich dem akuten hermeneutischen Problem zu stellen, dass die Apartheid aufwirft: Christliche Akteure stehen auf beiden Seiten einer gespaltenen Kirche und berufen sich auf die Bibel als Gottes Wort. Angesichts dessen muss gefragt werden, ob sthe Bible can be used for any purpose at all weil "both oppressor and oppressed claim loyalty to the same Church. ${ }^{49}$ Die Kairos-Theologen lösen diesen Konflikt nicht auf, fordern aber, dass Kritik an ihren eigenen Auslegungen und Argumentationen neben dem "biblical faith" auch die "Christian experience in South Africa ${ }^{50}$ berücksichtigen müsste. Damit expliziert das Dokument seinen kontextuellen Status und fordert dieses Eingeständnis auch von seinen Gegnern. Es scheint, als hätten die Kairos-Theologen die Ambivalenz der Bibel und ihrer Interpretationen erkannt und deswegen das zwar ebenso uneindeutige, aber doch präzisierende Kriterium der Erfahrung mit aufgenommen, das den Kontext ausdrücklich in den Blick nimmt.

\section{Die Grenze der Wahrhaftigkeit}

Beide Seiten der theologischen Apartheiddebatte können zum Teil als wahrhaftige Stimmen im Streit um die wahre Bibelauslegung gelten. Alle Dokumente, die den Apartheiddiskurs konstituieren, erwecken den Anschein, dass sie mehr oder weniger, aber jedenfalls überzeugt von dem sind, was sie schreiben. So findet Wahrhaftigkeit in der Interpretation ihre Grenze, wenn es darum geht, die ethische Verantwortlichkeit gegenüber dem Kontext des Subjekts der Interpretation sicherzustellen. Neben dem wichtigen Hinweis des Kairos-Dokuments, unbedingt den Erfahrungshorizont in der Produktion theologischer Aussagen zu berücksichtigen, also Theo-

\footnotetext{
${ }^{49}$ Kairos Theologen, Kairos Document: Challenge to the Church. A Theological Comment on the Political Crisis in South Africa, London 1985, 4.

${ }^{50}$ Kairos Document, 2. Besonders in dieser hermeneutischen Forderung wird die Verortung des Kairos-Dokuments in der Black Theology deutlich.Vgl. z.B. Itumeleng J. Mosala, Biblical Hermeneutics and Black Theology in South Africa, Grand Rapids, MI 1989.
} 
logie im Kontext zu treiben, möchte ich noch einen zweiten Aspekt hinzufügen. Dieser zweite Aspekt ist im Sinne einer konsequenten Haltung der Wahrhaftigkeit sogar geboten.

Bibelauslegungen, die nicht ein rein historisches oder philologisches, sondern auch theologisches und damit ein auf die Existenz des Menschen als Geschöpf Gottes in dieser Welt bezogenes Interesse aufweisen, müssen die Option zur Opposition gegen Aussagen der Bibel immer offen lassen. Denn biblische Texte können nicht per se als moralisch wertvoll gelten. Die Apartheid-Theologen waren nicht durchgehend schlechte Exegeten, die ihre Texte bis zur Unkenntlichkeit missbrauchten. Es gibt Narrative der Exklusion, Unterdrückung, Gewalt und Hegemonie in der Bibel, genauso wie Traditionen der Gleichberechtigung, der individuellen Würde und der Grenzen überwindenden Kommunikation und Liebe überliefert sind. ${ }^{51}$ Der südafrikanische Befreiungstheologe Itumeleng Mosala meint angesichts dessen:

"[T]he only adequate and honest explanation is that not all of the Bible is on the side of human rights or of oppressed and exploited people. Recognition of this is of vital importance for those who would use the Bible in the service of the struggle for the liberation of oppressed and exploited people. "52 $^{52}$

Dies anzuerkennen führt insbesondere reformatorische Theologien notwendigerweise in eine "Krise des Schriftprinzips, " wie sie schon mehrfach beschrieben worden ist. ${ }^{53}$ Und doch muss eine Theologie, die relevant, kritikfähig und nachvollziehbar sein will davon absehen, ethisch fragwürdige Texte verharmlosend zu deuten.Vielmehr muss sie auch dann wahrhaftig sein, wenn sie "texts of terror ${ }^{54}$ auslegt, indem sie sie als Teil der normativen Schrift der eigenen Tradition kritisiert.

- Moritz Gräper ist Vikar in der Evangelischen Kirche von Westfalen. Er wurde 2017 in Münster aufgrund seiner im Fach Neues Testament bei Hermut Löhr verfassten Arbeit »Bible and Apartheid» promoviert. Sein Hauptinteresse gilt Fragen der biblischen, interkulturellen und interreligiösen Hermeneutik.

${ }^{51}$ Vgl. "Identity Discourses in Early Christianity and Today« in Gräper, Bible and Apartheid.

${ }_{52}$ Mosala, Black Theology, 30.

${ }^{53}$ Vgl. z.B. Rochus Leonhardt/Martin Rösel, Reformatorisches Schriftprinzip und gegenwärtige Bibelauslegung. Ein interdisziplinärer Gesprächsbeitrag zur zeitgemäßen Schrifthermeneutik, Theologische Zeitschrift 56 (2000), 298-324.

${ }^{54}$ West, Gerald O., Towards an African Liberationist Queer Theological Pedagogy, Journal of Theology for Southern Africa 155 (2016), 216-223, hier 221. 